\title{
The influence of maternal and child FADS genotype on cord blood polyunsaturated fatty acid (PUFA) concentrations
}

\author{
Marie C. Conway $^{1}$, Maria S. Mulhern ${ }^{1}$, Emeir M. McSorley ${ }^{1}$, J.J. Strain ${ }^{1}$, Edwin van \\ Wijngaarden $^{2}$, Phil W. Davidson ${ }^{2}$, Gary J. Myers², Karin Wahlberg ${ }^{3}$, Conrad F. Shamlaye ${ }^{4}$, \\ Daniela Pineda ${ }^{3}$, Karin Broberg ${ }^{3}$ and Alison $\mathrm{J}_{\text {Yeates }}{ }^{1}$ \\ ${ }^{1}$ Nutrition Innovation Centre for Food and Health (NICHE), Ulster University, Coleraine, United Kingdom, \\ ${ }^{2}$ School of Medicine and Dentistry, University of Rochester, Rochester, USA, \\ ${ }^{3}$ The Laboratory of Medicine, Division of Occupational and Environmental Medicine, Lund University, Lund, Sweden \\ and \\ ${ }^{4}$ Ministry of Health, Mahé, Seychelles
}

\begin{abstract}
Optimal maternal polyunsaturated fatty acid (PUFA) status is essential for foetal development. The desaturase enzymes, encoded by the fatty acid desaturase (FADS) genes, are involved in the endogenous synthesis of long chain (LC)PUFA and influence maternal LCPUFA concentrations. The minor allele of various FADS SNPs has been associated with increased maternal concentrations of the precursors linoleic acid (LA) and $\alpha$-linolenic acid (ALA), and lower concentrations of the LCPUFA arachidonic acid (AA) and docosahexaenoic acid (DHA); however, there is limited research to date on the influence of FADS genotype on cord PUFA status. The aim of the current study was to investigate the influence of maternal and child genetic variation on cord blood PUFA status in a high fish-eating cohort.

Cord blood samples collected from mother-child pairs $(\mathrm{n}=1088)$ taking part in the Seychelles Child Development Study $(\mathrm{SCDS})$ Nutrition Cohort 2 (NC2) were analysed for total serum PUFA. Maternal $(n=1088)$ and child genotype $(n=592)$ were determined for the FADS SNPs rs174537, rs174561, rs174575, and rs3834458. Regression analysis determined associations between maternal and child FADS genotype and cord PUFA status. In all regression models, the major allele homozygote genotype for each SNP was used as the reference group.

Directions of significant associations were as predicted. In mothers, the minor allele homozygote genotype for SNPs rs174537, rs174561 and rs3834458 was associated with lower cord DHA and lower total n-3 PUFA when compared to the major allele homozygous genotype $(\mathrm{p}<0.05$ for all). The heterozygous genotype was associated with increased concentrations of LA compared to the reference genotype for $\mathrm{rs} 174561(\mathrm{p}=0.021)$ and $\mathrm{rs} 383448(\mathrm{p}=0.023)$. In children, the heterozygous genotype was associated with lower AA concentrations and lower cord n-6:n-3 ratio for all SNPs ( $<<0.05$ for all) compared to those with the major allele homozygous genotype. A lower cord AA:LA ratio was also observed for children heterozygous for rs174547, rs174561 and rs174575 (p< 0.05 for all). Contrary to expected, there were no associations between cord PUFA concentrations and child minor allele homozygous genotype.

The current study indicates that variation in maternal and child FADS genotype influences cord PUFA concentrations, despite the high intake of preformed dietary LCPUFA from fish in this population. The sample size for minor allele homozygous children was likely too small to observe any statistically significant associations in the current analysis. Further research is needed to determine whether increased dietary intake can compensate for lower PUFA status as a result of FADS genotype.
\end{abstract}

\section{Conflict of Interest}

There is no conflict of interest 